\title{
Clival Metastasis of Hepatocellular Carcinoma with an Initial Histopathological Diagnosis of Atypical Pituitary Adenoma
}

\author{
Hiroyuki Mishima ${ }^{1}$ Junichi Ayabe ${ }^{1}$ Masahide Watanabe ${ }^{1}$ Sota Togin $^{1}$ Yuusuke Tsuchiya ${ }^{1}$ \\ Taisuke Kawasaki ${ }^{1}$ Naoyuki Noda ${ }^{1}$ Takumi Maruyama ${ }^{1}$ Yoshihide Tanaka ${ }^{1}$ \\ ${ }^{1}$ Department of Neurosurgery, Yokosukakyousai Hospital, \\ Yokosukashi, Kanagawaken, Japan \\ J Neurol Surg Rep 2019;80:e18-e22. \\ Address for correspondence Hiroyuki Mishima, MD, Department of \\ Neurosurgery, Yokosukakyousai Hospital, 1-16 Yonegahama Street, \\ Yokosukashi, Kanagawaken 238-8558, Japan \\ (e-mail: mishima0521@gmail.com).
}

\author{
Abstract \\ Keywords \\ - clival tumor \\ - hepatocellular \\ carcinoma \\ - ectopic pituitary \\ adenoma \\ - $\alpha$-fetoprotein \\ - cancer of unknown \\ primary site
}

Histologically, hepatocellular carcinoma (HCC) and atypical pituitary adenoma are similar, posing a potential clinical problem. A 76-year-old woman, whose past medical history was significant for hepatitis $\mathrm{C}$ virus $(\mathrm{HCV})$, positivity without liver cirrhosis was presented with abducens nerve $(\mathrm{CN} \mathrm{VI)}$ palsy. Contrast-enhanced magnetic resonance imaging (MRI) showed a clival tumor with infiltration of the right cavernous sinus. Subtotal resection was performed using a right anterior petrosal approach. Histological diagnosis was pituitary adenoma. Thirty-four days after the operation, MRI imaging showed residual tumor growth without any liver masses on computed tomography (CT). The patient underwent a second operation using an endoscopic transnasal transsphenoidal approach. Histological examination of the specimen gave a positive reaction for $\alpha$-fetoprotein. Repeat whole-body CT showed an enhanced liver mass. Based on these findings, the lesion was diagnosed as metastatic HCC. The patient died of liver failure 6 months later. In this case, tumor diagnosis was difficult because of the histological similarity of HCC and pituitary adenoma, and the fact that the clival tumor grew prior to presentation of the liver mass on CT. This case emphasizes the importance of comparing the pathological diagnosis with the patient's clinical course.

\section{Introduction}

Liver cancer is sometimes called a silent killer because some patients have little to no early signs or symptoms. At times, metastatic lesions are found before the primary lesion is diagnosed. In this case, the tumor was first diagnosed as an atypical pituitary adenoma after the patient's first operation, then grew rapidly and without any evidence of liver tumor on computed tomography (CT). Following a second operation, we diagnosed the tumor as a clival metastasis of hepatocellular carcinoma (HCC).

\section{Case Report}

The patient was a 76-year-old female, whose past medical history was significant for hepatitis C virus (HCV), positivity

received

November 15, 2018

accepted

DOI https://doi.org/

10.1055/s-0039-1685207.

ISSN 2193-6358. without liver cirrhosis. She presented with right abducens nerve ( $\mathrm{CN} \mathrm{VI}$ ) palsy. Contrast-enhanced magnetic resonance imaging (MRI) and CT scan showed a clival tumor with osteolysis and infiltration of the right cavernous sinus (-Figs. 1 and 2). All laboratory data were within normal limits, including liver function. We removed the tumor using the right anterior petrosal approach. The tumor was hemorrhagic, so we finished this operation as a subtotal removal. The specimen was negative for adrenocorticotropic hormone, growth hormone, prolactin, thyroid-stimulating hormone, and folliclestimulating hormone/luteinizing hormone. The tumor was pathologically diagnosed as a pituitary adenoma by three different pathologists ( - Fig. 3 ). The diagnosis was ultimately determined to be ectopic pituitary adenoma. Thirty-four days after the operation, the patient presented at our hospital

\footnotetext{
(c) 2019 Georg Thieme Verlag KG Stuttgart · New York
}

License terms

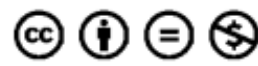



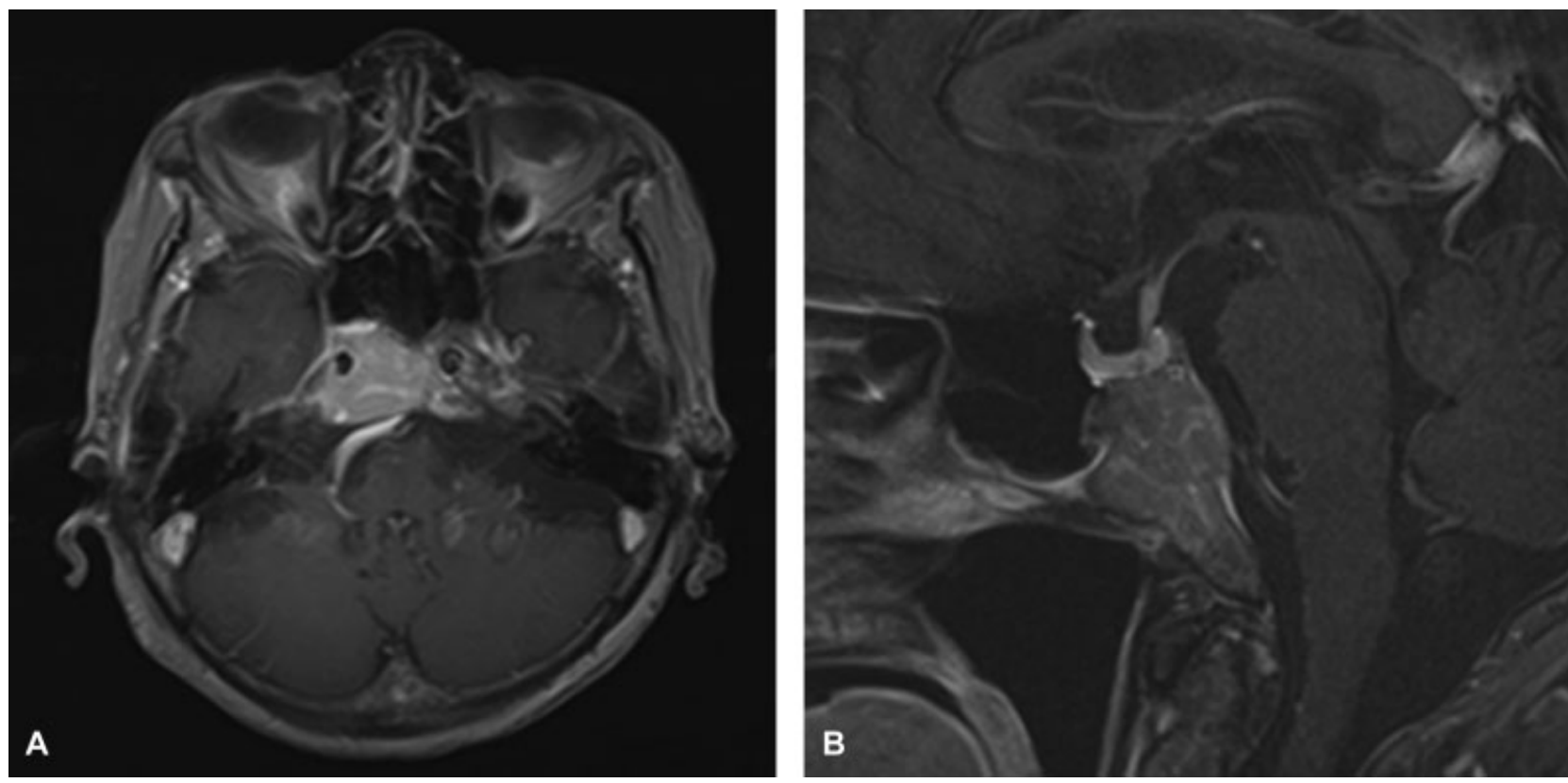

Fig. 1 Preoperative MRI, with contrast axial (A) and sagittal (B) sections demonstrating a contrast-enhanced clival tumor. MRI, magnetic resonance imaging.

complaining of massive bleeding from her nose. She had difficulty breathing, so she was intubated within the outpatient clinic. MRI showed significant growth of the residual clival tumor (-Fig. 4) and pathological diagnosis, so the patient was taken for contrast-enhanced whole-body CT, which showed no evidence of tumor, including in the liver. (-Fig. 5). We removed the tumor using an endoscopic transnasal transsphenoidal approach. The tumor bled massively during surgery, necessitating the transfusion of $3,600 \mathrm{~mL}$ of red blood cells (RBCs), $720 \mathrm{~mL}$ of fresh frozen plasma, and $400 \mathrm{~mL}$ of platelets. Contrast-enhanced whole-body CT scan-

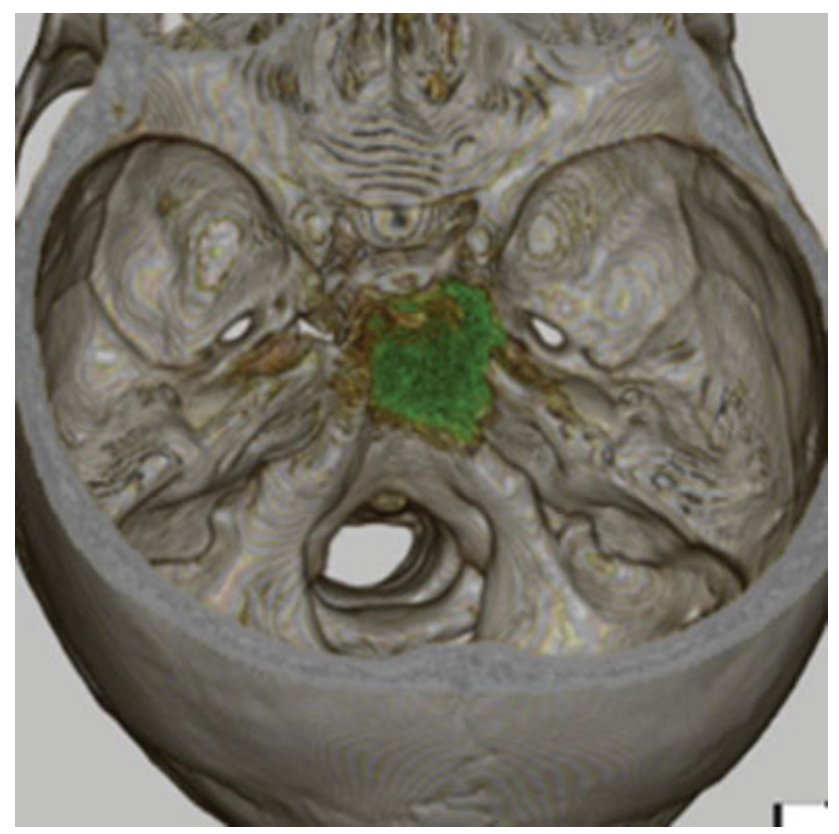

Fig. 2 Preoperative three-dimensional CT with contrast showing the clival tumor extending laterally from the right cavernous sinus into the petrous apex. CT, computed tomography. ning was performed again 60 days after the first operation and it showed a new enhanced liver mass (-Fig. 6). Blood tests revealed significant elevation of $\alpha$-fetoprotein (AFP), and protein induced by the absence of vitamin $\mathrm{K}$ or antagonists-II. Immunostaining of the pathological specimen was positive for AFP ( - Fig. 7). $\mathrm{K}_{i}-67$ expression of the specimen was $20 \%$, and it was the same in the first specimen. We determined that the clival tumor was actually metastatic HCC. The residual tumor rapidly increased in size following the second surgery

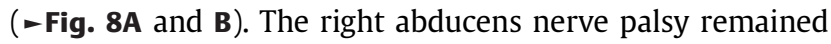
unchanged during the course, whereas the left abducens nerve

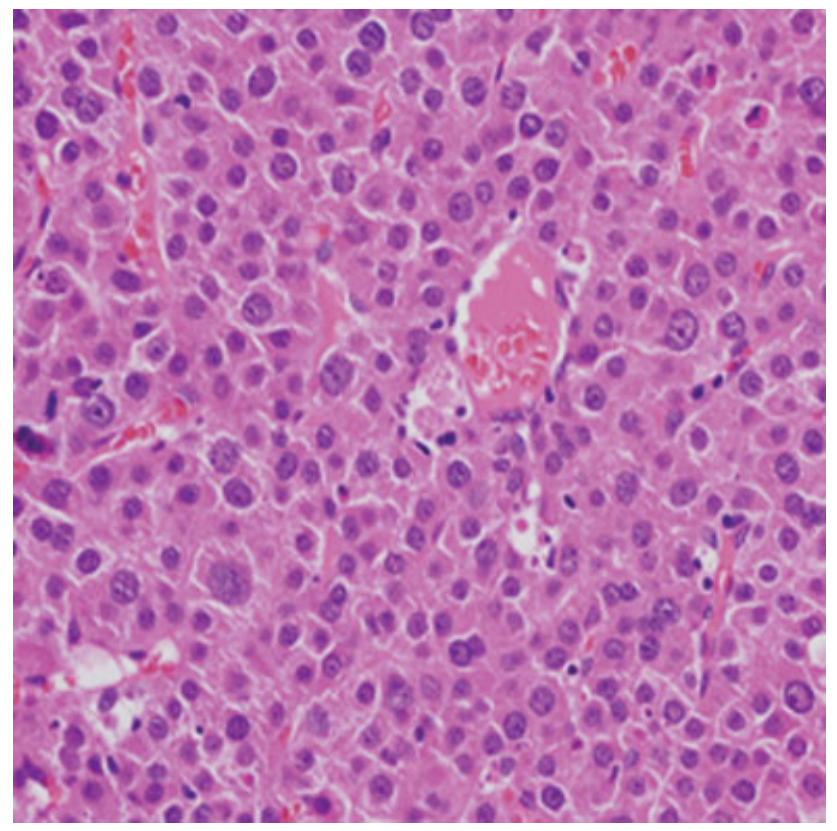

Fig. 3 Pathologic evaluation at $\times 40$ magnification. The histologic section demonstrated anisonucleosis, sheet-like proliferation, eosinophilic cytoplasm, and mitosis. 


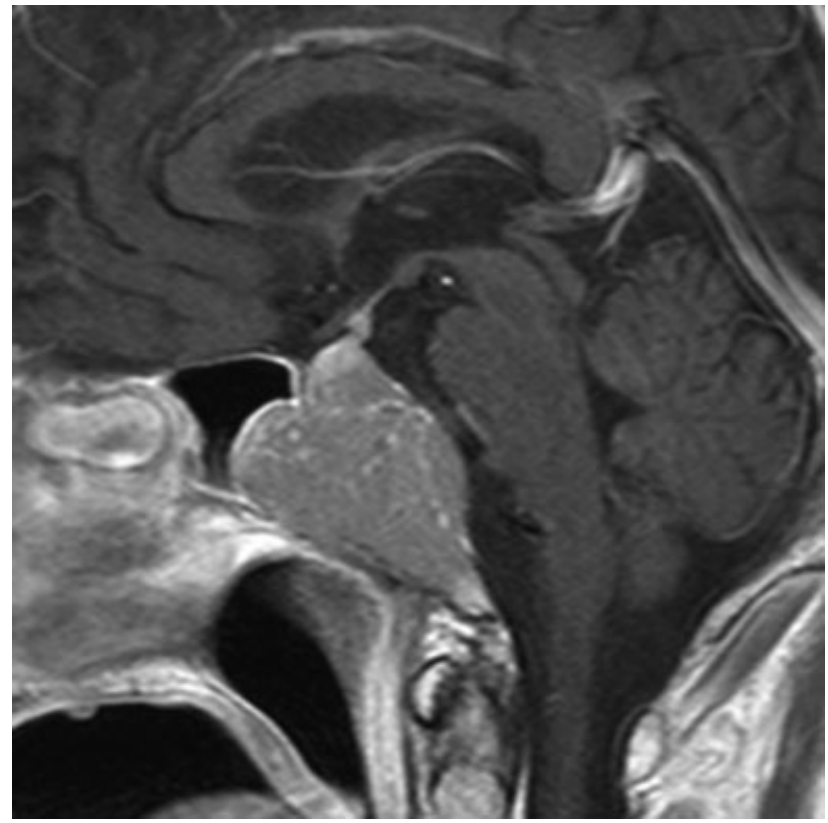

Fig. 4 MRI 34 days after the first operation with a contrast sagittal section, demonstrating significant tumor growth. MRI, magnetic resonance imaging.

palsy appeared 2 weeks following the second operation. The patient died of liver failure 6 months after the first operation.

\section{Discussion}

The pathologic similarity of HCC and atypical pituitary adenoma, as well as the fact that the clival metastasis tumor grew before the CT scan revealed liver HCC, complicated the diagnostic process in this patient. Our patient's tumor was initially diagnosed as an ectopic pituitary adenoma. Histo-

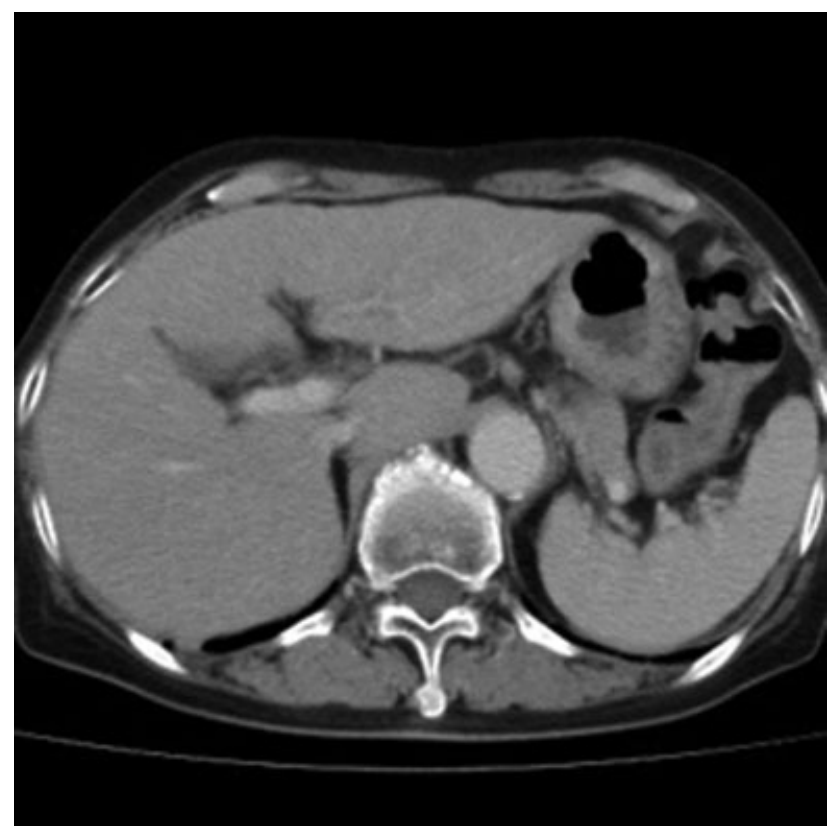

Fig. 5 Noncontrast whole-body CT imaging 1 day before the second operation did not reveal any mass, including in the liver. CT, computed tomography.

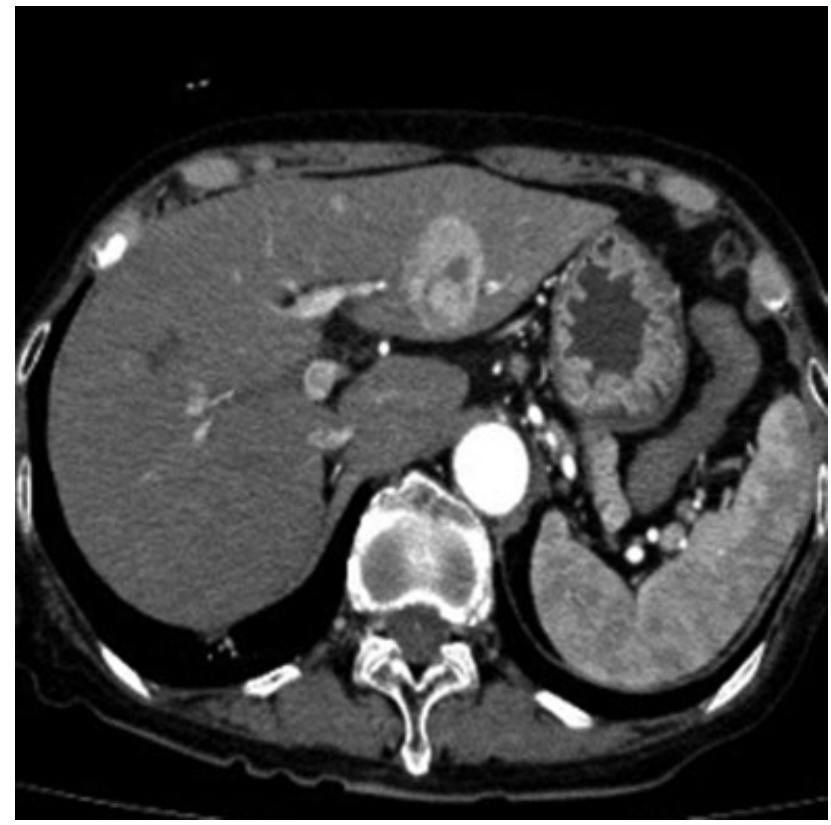

Fig. 6 Contrast-enhanced whole-body CT scanning after the second operation showed an enhanced liver mass. CT, computed tomography.

pathologic appearance of HCC varies greatly from patient to patient. The most common histologic growth patterns include trabecular-resembling normal liver tissue, pseudoglandular, or acinar with possible bile or fibrin content, and solid. ${ }^{1}$ Solid-type HCC could closely resemble pituitary adenoma on pathological imaging. A side-by-side comparison of pathological images of a solid-type HCC and a diffuse-type pituitary adenoma is presented in - Table $1 .^{2-4}$ HCC and pituitary adenomas are distinguishable via immunostaining, such as hepatocyte and AFP but immunostaining is only performed when it is considered necessary.

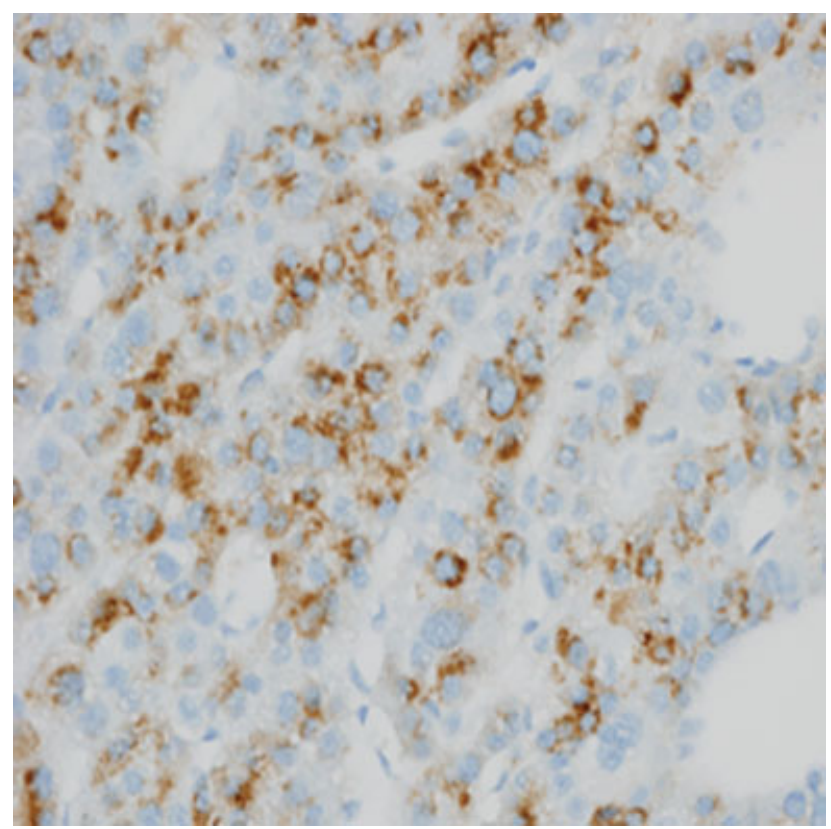

Fig. 7 During immunostaining, the pathological specimen was AFP positive. AFP, $\alpha$-fetoprotein. 

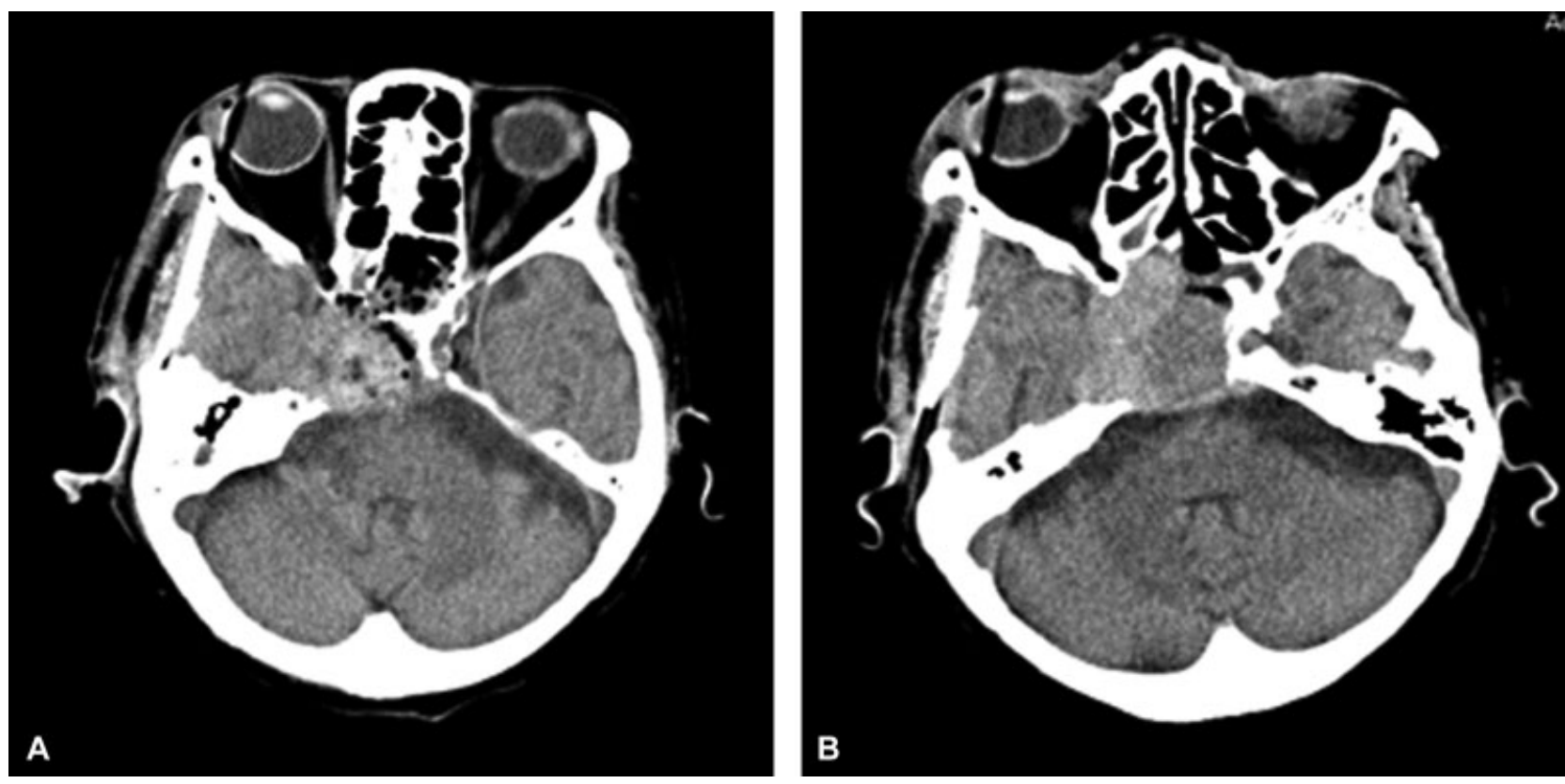

Fig. 8 CT immediately after the second operation (A) and one month after the second operation (B). CT, computed tomography.

Table 1 A histopathological image comparison of HCC (solid-type) and pituitary adenoma (diffuse-type)

\begin{tabular}{|l|l|l|}
\hline & Solid-type HCC & Diffuse-type pituitary adenoma \\
\hline Morphologic features & $\begin{array}{l}\text { Diffuse proliferation } \\
\text { Trabecular arrangement } \\
\text { Inconspicuous sinusoids }\end{array}$ & $\begin{array}{l}\text { Diffuse proliferation } \\
\text { Fibrous component in the stroma } \\
\text { Formation of new blood vessels }\end{array}$ \\
\hline Hematoxylin and eosin stain & Eosinophilic & Eosinophilic basophilic \\
\hline $\mathrm{K}_{i} 67$ & $0.1-89 \%$ & $1-23 \%$ \\
\hline Anisonucleosis & $(+)$ & $(+\sim-)$ \\
\hline Mitosis & $(+)$ & $(+\sim-)$ \\
\hline Immunostaining & Hepatocyte, AFP & PRL, ACTH, TSH, GH, none \\
\hline
\end{tabular}

Abbreviations: ACTH, adrenocorticotropic hormone; AFP, $\alpha$-fetoprotein; GH, growth hormone; HCC, hepatocellular carcinoma; PRL, prolactin; TSH, thyroid-stimulating hormone.

When we operated on this patient the second time, the CT scan showed no liver masses. Metastatic tumors diagnosed prior to primary tumors are called cancer of unknown primary site (CUP). CUP accounts for 3 to $5 \%$ of all tumor diagnoses. Eighty percent of all patients diagnosed with CUP have a poor prognosis and a median overall survival of 6 months. It is possible that the progression of CUP parallels the primary tumor, and CUP metastases are an early event in tumor development. ${ }^{5}$ In this case, the liver tumor grew rapidly over a short time, suggesting that the tumor was highly malignant. The clival metastasis was an early HCC progression event, and the metastasis grew more rapidly than the primary site. When cancer is diagnosed as CUP, repeat $\mathrm{CT}$, positron emission tomography, and serum tumor markers are recommended to find the true primary cancer. ${ }^{5}$

Ectopic pituitary adenoma was the suspected diagnosis after the first operation. The location of the tumor itself did not exclude a diagnosis of ectopic pituitary adenoma. Karras et al reported an ectopic pituitary adenoma that involved the entire clivus, laterally extended to the petroclival junction, and bilaterally invaded the cavernous sinuses, with further bilateral encasement of the internal carotid arteries without direct extension into the sella. ${ }^{6}$ The clinical course of this case is quite different from that of a patient with ectopic pituitary adenoma. First, we encountered massive bleeding from the tumor during each operation, and during the second operation the patient needed transfusions. Second, there was rapid tumor regrowth. The size of the residual tumor doubled in approximately 1 month. Third, the patient demonstrated spontaneous and idiopathic hemorrhaging from her nose. The volume of the hemorrhage was so large that the patient required intubation. Typically, patients with ectopic pituitary adenomas present as asymptomatic or with focal neurological deficits. ${ }^{6}$ The clinical course of this patient indicated the tumor was aggressive and could be malignant.

\section{Conclusion}

Solid-type HCC can appear pathologically similar to a pituitary adenoma. It is difficult to diagnose a clival metastasis of 
e22 Metastasis of HCC with an Initial Diagnosis of Pituitary Adenoma Mishima et al.

HCC from preoperative images if the tumor is a potential CUP. When we doubt a histopathological diagnosis, it is important to compare the pathological diagnosis with the patient's clinical course.

\section{Conflict of Interest}

None.

\section{References}

1 Schlageter M, Terracciano LM, D’Angelo S, Sorrentino P. Histopathology of hepatocellular carcinoma. World J Gastroenterol 2014;20(43):15955-15964
2 King K-L, Hwang J-J, Chau G-Y, et al. Ki-67 expression as a prognostic marker in patients with hepatocellular carcinoma. J Gastroenterol Hepatol 1998;13(03):273-279

3 Wakabayashi T, Shibui S, Hirose T, Kobayashi T. Color atlas of brain tumor pathology. 4th edition. Tokyo: Igaku-Syoin LTD.; 2017: 163-166

4 Hirohata T, Ishii Y, Matsuno A. Atypical pituitary adenoma and pituitary carcinoma. Endocrinology Diabetology Metabolism 2015;40(05):345-350

5 Losa F, Soler G, Casado A, et al. SEOM clinical guideline on unknown primary cancer (2017). Clin Transl Oncol 2018;20 (01):1-8

6 Karras CL, Abecassis IJ, Abecassis ZA, et al. Clival ectopic pituitary adenoma mimicking a chordoma: case report and review of the literature. Case Rep Neurol Med 2016;2016:8371697 